\title{
Analysis of Risk Diversification --Based on Bitcoin
}

\author{
Junchao $\mathrm{Xia}^{1, *}$ \\ ${ }^{1}$ Chongqing BI Academy, Chongqing, 401120, China \\ *Corresponding author.Email: eric.xia@biacademy.cn
}

\begin{abstract}
Bitcoin is an essential component in the cryptocurrency system and plays a vital role in the economic system. This paper uses analytical methods to conduct in-depth research on Bitcoin. I will explain their opinions about its ability to invest and risk diversification, and then we try to verify some conclusions of the MPT model. First, my research shows that based on the modern portfolio theory, the risks of bitcoin could be diversified with some trading assets on the actual market. Second, two essential risks of Bitcoin are the legality and the cyber risk. On the one hand, the research in this article provides a theoretical speech on Bitcoin. On the other hand, it provides theoretical suggestions for the application and development of Bitcoin.
\end{abstract}

Keywords: Bitcoin, cryptocurrency, MPT, risk

\section{INTRODUCTION}

Bitcoin has been viewed as an investable product because of its sharp price growth. It took more than ten years to go from less than a few cents to sixty thousand dollars. Therefore, many investors reckon that they could make much money by investing in bitcoins. In April and May of this year, countless people began to mine due to the sharp rise in the price of Bitcoin. Same as the Gold Rush in the U.S. in $18 \mathrm{TH}$ century, the price of high-end graphics cards has risen a lot.

Bitcoin is an essential component in the cryptocurrency system and plays a vital role in the economic system. Nakamoto first introduced Bitcoin in 2008 in the form of peer-to-peer digital currency. Peer-to-peer transmission means a decentralized payment system. The most salient characteristic is that Bitcoin does not rely on specific currency institutions to issue, unlike traditional currencies. The only way to produce Bitcoin is through extensive calculations based on specific algorithms, known as "mining." Therefore, mining Bitcoin can be considered as a reward for getting an excellent answer to a puzzle. The calculation of network nodes generates Bitcoin. Anyone can participate in manufacturing Bitcoin that can be circulated all over the world. It can be bought and sold on any computer connected to the Internet. Anyone can mine, buy, sell or collect Bitcoin from any location, and outsiders cannot identify the user's identity information during the transaction.
Economists have polarized attitudes towards Bitcoins. Economists of the Keynesian school believe that Bitcoin's fixed aggregate currency sacrifices its controllability, and it will inevitably lead to deflation and harm the overall economy. Paul Krugman stated that crypto-assets such as Bitcoin are a Ponzi scam. The Austrian school of economists holds the opposite view. They believe that the less government intervention in currency, the better. Deflation caused by a fixed amount of money is not a big deal, and it is even a sign of social progress.

Besides some economists in the world, several cohorts studied analyses have examined the function of Bitcoin. Wang, Tang, Xie, and Chen (2019) shown that Bitcoin acts as a hedge for stocks, bonds, and SHIBOR (Shanghai Interbank Offer Rate) and is a safe haven when extreme price changes occur in the monetary market [1]. Kristoufek (2020) discussed the correlation between Bitcoin, S\&P500, and VIX. They compared gold with Bitcoin as well, suggesting that Bitcoin was unsubstantial and its proposed role as a safe haven was far-fetched. On the other hand, gold came out as a clear winner compared to Bitcoin. Nevertheless, Kristoufek (2020) found strange phenomena in the COVID-19 period. Bitcoin started to become a possible safe haven asset to prevent financial catastrophes [2].

A large and growing body of literature has investigated the characteristics and functionality of Bitcoin and other types of cryptocurrencies. Bitcoin has a short history compared with fiat currency like the U.S. 
dollar or Chinese Yuan. Nakamoto (2008) created Bitcoin. He firmly believes that Bitcoin is the peer-to-peer version of electronic cash and a cost-efficient way to transact because Bitcoin can work without a financial intermediary [3].

During the Covid-19 era, much more information has become available on whether Bitcoin or any other cryptocurrencies can be used as hedging instruments and a safe haven for the capital market. Surveys such as those conducted by Yuhanitha and Robiyanto (2021) have shown that Bitcoin can act as a hedge for the Indonesian capital market in the Covid-19 pandemic era. Still, Bitcoin cannot be the safe haven for the Indonesian capital market in the Covid-19 pandemic era[4]. Also, Yuhanitha and Robiyanto (2021) find that the CSPI (Composite Stock Price Index) price began to rise slightly, and the movements were relatively stable. On the same date, it also showed that cryptocurrencies have the opposite cycle movements or negative correlations from the CSPI price. Therefore, when the CSPI's movements strengthen, the price movements of cryptocurrencies weaken [4]. In addition, Nguyen, Bodisco, and Thaver (2018) draw our attention to other factors that affect the price of Bitcoin. They found that Bitcoin's price is driven by transactional demand, such as the supply of Bitcoin or the size of the Bitcoin economy, and speculative demand, namely media attention or prices of other cryptocurrencies [5].

Many scholars also find that Bitcoin could be a haven during extreme periods. For example, in the COVID-19 period, some scholars found that Bitcoin will be affected by the market sentiment, which gave them the idea of whether bitcoin could be used as a financial tool in hedging. Meanwhile, some professionals put forward that the market risks of bitcoin are a little bit different from our regular hedge tools. Because of its unique characteristics, its price fluctuations may be easily affected by investors' behaviours.

One paper mentioned that bitcoin plays different roles in different areas. For example, in a developing country, Bitcoins could be used as a strong hedge for investors. On the contrary, it effectively diversifies developed countries' markets, regional indices, and commodities for investors. In addition, Bitcoin is also a safe place for some national equity indices, regional indices, and commodities.

To sum up, based on the above studies, we can find that research on Bitcoin mainly exist in function, while there are few types of research on risk. Therefore, we will discuss the external risks, cyber risks, and market risks of Bitcoin. In addition, we will show that the risk of Bitcoin can be divided by using some portfolios.

\section{DATA AND METHOD}

\subsection{Data}

We use the Bitcoin market price in USD (Code: BTC), the S\&P 500 index (Code: SPX), the 3-month U.S. Treasury Bond Rate (Code: FBTB3M), the Thomson Reuters/Core Commodity index (Code: CRB), and S\&P 500 Investment Grade Corporate Bond Index (Code: SP5IGBIT) as our portfolio components. We captured the data from Bloomberg and selected the weekly data for the period of $08 / 18 / 2017$ to $07 / 30 / 2021$. The statistic indicators will be listed in Table.1.

Table 1. Statistic indicators of 5 indexes

\begin{tabular}{|c|cc|}
\hline Index & Mean & Standard deviation \\
\hline FBTB3M & -0.1436 & 0.1596 \\
Bitcoin & 0.9287 & 0.8308 \\
SPX & 0.1710 & 0.1976 \\
CRB & 0.0658 & 0.0722 \\
SP5IGBIT & 0.0566 & 0.0695 \\
\hline
\end{tabular}

\subsection{Method}

I will use the MPT model to try to verify the result above.

MPT (Modern portfolio theory) is the basic framework in our project. Modern portfolio theory (MPT) is a theory on how risk-averse investors can construct portfolios to maximize expected returns based on a given level of market risk. In his paper "Portfolio Selection," Harry Markowitz pioneered this theory, published in the Journal of Finance in 1952. He was later awarded a Nobel Prize for his work on modern portfolio theory[6]. After Harry Markowitz won the Nobel Prize, other economists have attribution towards the MPT. Myles E. Mangram tries to simplify the MPT by suggesting efficient computer-based 'short-cuts' to these performing these intricate calculations[7]. The paper of Omisore, I., Yusuf, M., \& Christopher, N. (2011) shows the relevance of the modern portfolio theory as an investment portfolio tool in portfolio decision making[8].

MPT theory will research the relationship between standard deviation $\sigma$ and the expected return $R$. With the combination of different components in the portfolios, the return and standard deviation will be described as

$$
\begin{gathered}
\text { (2) } R_{P}=\omega^{T} R_{\text {equity }}=\sum_{i=1}^{n} \omega_{i} R_{i} \\
\text { (3) } \sigma_{P}^{2}=\omega^{T} \sum \omega=\sum_{i=1}^{n} \sum_{j=1}^{n} \omega_{i} \omega_{j} \operatorname{cov}\left(R_{i}, R_{j}\right)
\end{gathered}
$$

Then we define the parameters in the above, $\omega$ is a $n \times 1$ vector which means the weights of each equity in 
the portfolio, $\omega_{i}$ means the $i$ th component in the portfolio, and we will have the $\sum \omega_{i}=1 . R_{\text {stocks }}$ stands for a $1 \times n$ vector which is composed by the return of each included component. $\Sigma$ means the covariance matrix of the equities in the portfolio.

After we have the Efficient frontier, we may consider adding the risk-free assets into our portfolio, the CML (Capital Market Line) and CAL (Capital Allocation Line). The return of a risk-free asset will be located on the point $(0, R)$, which means the risk of these assets is 0 , and we can connect these points with any other points on the efficient frontier. The straight line is CAL (Capital Allocation Line). And we define the slope as sharp ratio

$$
\text { (1)Sharpe Ratio }=\frac{R_{p}-R_{f}}{\sigma_{p}}
$$

We could find the line tangent to the efficient frontier, where we may have the maximized Sharp Ratio, and we call this line CML(Capital Market Line).

Sharp Ratio provides us a target to measure the price of the unite of risk. A higher Sharp Ratio means a higher return when we are going to take the risk; also, we could say we may suffer from lower risk at the same return lever when we have the higher sharp Ratio. Some scholars also studied the Sharp Ratio in the portfolio. Bailey, D. H., \& Lopez de Prado, M. analysis the Sharp Ratio with Python [9]. And Sharpe, W. F. is the first one to define Sharpe Ration and apply it to different fields [10].

\section{RESULT AND DISCUSSION}

In this part, we will explain their opinions about its ability to invest and risk diversification, and then we will try to verify some conclusions with the MPT model.

\subsection{Steps and analysis}

In this project, we follow the following steps to generate the efficient frontier:

Step 1: Download weekly market data from Bloomberg.

Step 2: Data cleaning (including check the N.A. and abnormal values, match the time).

Step 3: Calculate the returns of the indexes.

Step 4: calculate the mean, standard deviation, covariance, and correlation coefficient.

Step 5: use the random function to generate weights of portfolios and standardization.

Step 6: plot the portfolios and fine the efficient portfolio that satisfies our requirements.

Based on the MPT model, we created a portfolio of 5 indexes.

And the covariance matrix:

Table 2. The covariance matrix of 5 indexes

\begin{tabular}{|c|ccccc|}
\hline Indexes & FBTB3M & Bitcoin & SPX & CRB & SP5IGBIT \\
\hline FBTB3M & 1.0000 & -0.0260 & -0.0108 & 0.1519 & -0.1944 \\
Bitcoin & -0.0260 & 1.0000 & 0.1761 & 0.1677 & 0.0928 \\
SPX & -0.0108 & 0.1761 & 1.0000 & 0.3074 & 0.4233 \\
CRB & 0.1519 & 0.1677 & 0.0374 & 1.0000 & 0.1426 \\
SP5IGBIT & -0.1944 & 0.0928 & 0.4233 & 0.1426 & 1.0000 \\
\hline
\end{tabular}

From Table 1, we can conclude that Bitcoin is a high-risk and high-return investment product. That is because its price rises too fast. It only took more than ten years to go from less than a few cents to sixty thousand dollars. Therefore, many people earn money through "mining" and transaction of Bitcoin. Through the analysis of statistics from 2017-08-18 to 2021-07-30, we know that the mean return of bitcoin is 0.9287 , and its standard deviation is 0.8308 . Hence, it is clear to say the bitcoin is a high-risk and high-return investment product. Also, we made a plot of Bitcoin return in Figure 1 to easily show Bitcoin's entire return. 


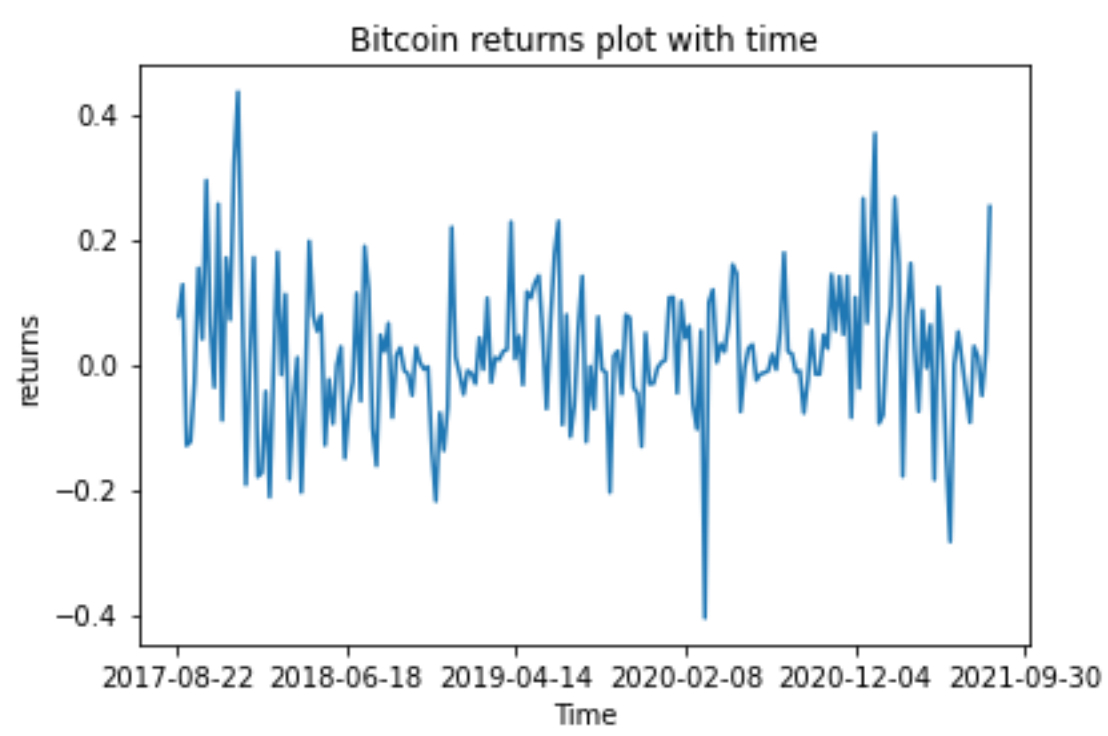

Figure 1. Return Plot of Bitcoin in USD from 08/18/2017 to 07/30/2021

Based on the idea of MPT, our next step is to prove that bitcoin could diversify the risks of the market portfolio. Our real financial market was mainly composed of commodity trading, equity trading, and Fixed income trading. That is why we choose such five indexes above as our portfolio. Thus, our portfolio could be a relatively comprehensive one to reflect the situation in a real market.

We first draw the efficient frontier of the 5 indexes and have the following plot in Figure 2.

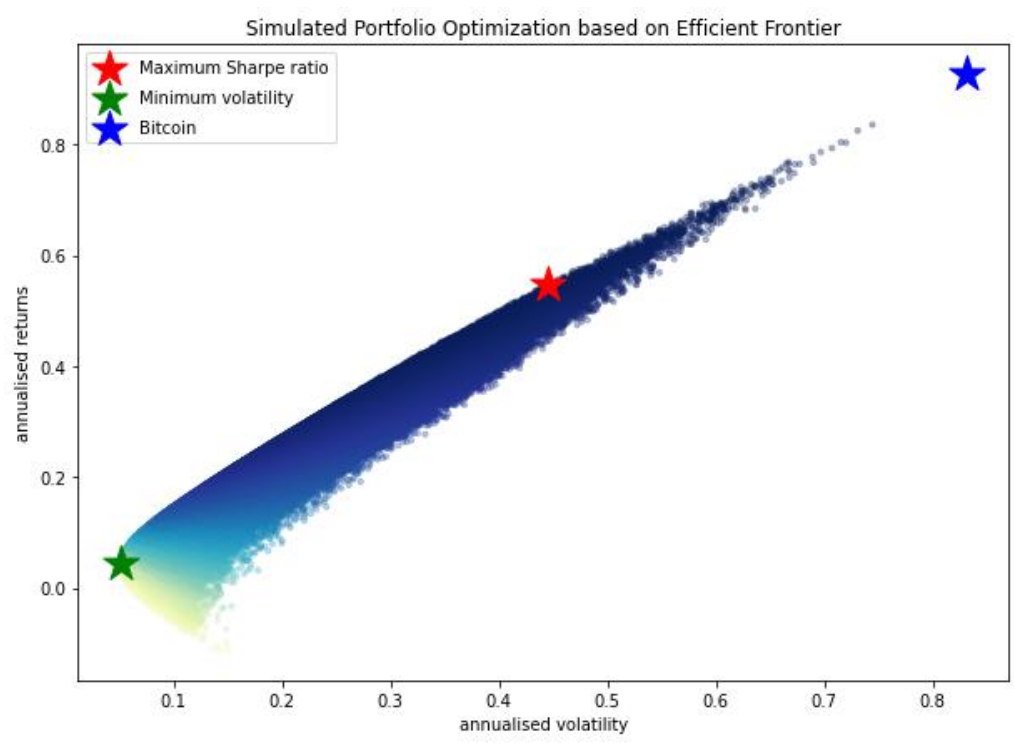

Figure 2. Plot of Efficient Frontier with bitcoin

We totally simulated 500,000 possible weights of portfolios and have the above plot. Because of the High risk and High return of Bitcoin, the plot is stretched very long. Meanwhile, we also created another portfolio without Bitcoin and plotted the Efficient Frontier, which seems to be much fatter. We can see the plot in Figure3. 


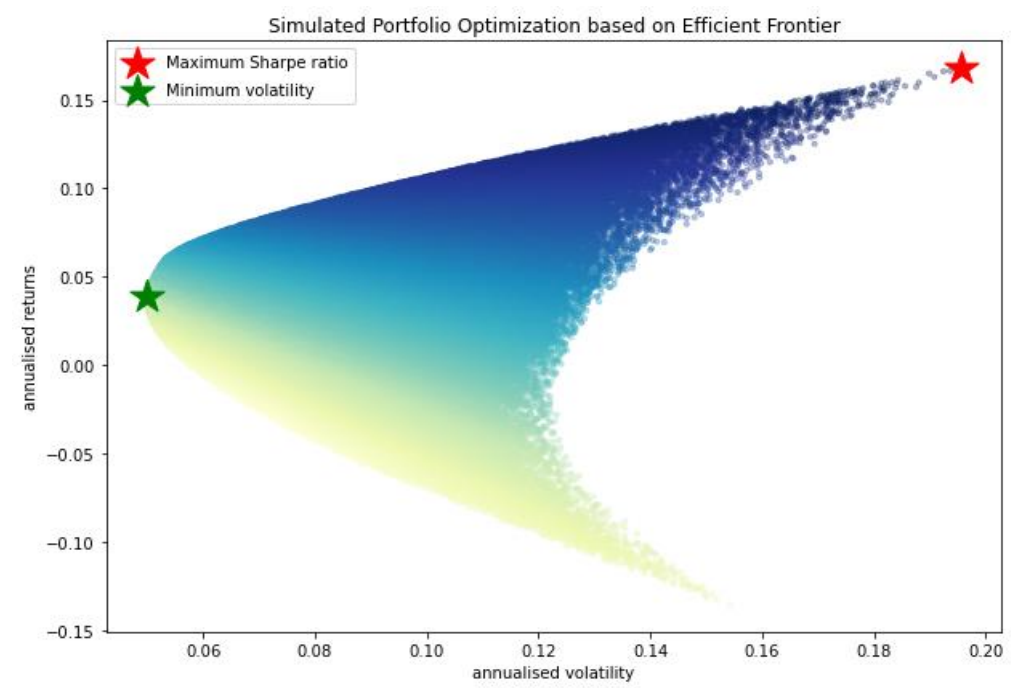

Figure 3. Plot of Efficient Frontier without bitcoin

Then, we will compare these two portfolios' Sharp Ratio at the same specific standard deviation. Sharp Ratio stands for the return of unit risk; a higher sharp
Ratio means the lower risk cost. Here are the sheets which show us the sharp ratios under specific standard deviations:

Table 3. Portfolio with Bitcoin at specific standard deviation

\begin{tabular}{|c|c|c|c|c|c|c|c|c|}
\hline & Number & 1 & 2 & 3 & 4 & 5 & 6 & 7 \\
\hline \multirow[t]{5}{*}{ weight } & FBTB3M(\%) & 77.21 & 71.23 & 73.48 & 66.18 & 65.11 & 64.05 & 58.09 \\
\hline & Bitcoin $(\%)$ & 17.04 & 18.73 & 22.74 & 23.63 & 26.35 & 27.73 & 34.91 \\
\hline & SPX $(\%)$ & 4.94 & 1.74 & 1.05 & 0.41 & 3.04 & 3.84 & 3.45 \\
\hline & CRB(\%) & 0.21 & 6.34 & 2.42 & 9.63 & 4.14 & 1.14 & 2.91 \\
\hline & SP5IGBIT(\%) & 0.60 & 1.96 & 0.31 & 0.16 & 1.36 & 3.23 & 0.63 \\
\hline \multirow[t]{3}{*}{ Indicators } & Mean(\%) & 0.19 & 0.19 & 0.22 & 0.23 & 0.24 & 0.25 & 0.28 \\
\hline & Standard Deviation & 0.05 & 0.07 & 0.10 & 0.13 & 0.15 & 0.17 & 0.20 \\
\hline & Sharp ratio(\%) & 3.53 & 2.60 & 2.11 & 1.63 & 1.54 & 1.42 & 1.36 \\
\hline
\end{tabular}

Table 4. Portfolio without Bitcoin at specific standard deviation

\begin{tabular}{|c|c|c|c|c|c|c|c|c|}
\hline & Number & 1 & 2 & 3 & 4 & 5 & 6 & 7 \\
\hline \multirow[t]{4}{*}{ weight } & FBTB3M(\%) & 34.60 & 28.45 & 20.09 & 9.04 & 2.39 & 0.25 & 0.46 \\
\hline & $\operatorname{SPX}(\%)$ & 62.89 & 70.72 & 79.63 & 88.66 & 95.03 & 97.06 & 99.10 \\
\hline & $\mathrm{CRB}(\%)$ & 2.12 & 0.83 & 0.27 & 0.37 & 1.59 & 0.00 & 0.25 \\
\hline & SP5IGBIT(\%) & 0.38 & 0.01 & 0.01 & 1.93 & 0.99 & 2.69 & 0.19 \\
\hline \multirow[t]{3}{*}{ Indicators } & Mean(\%) & 0.14 & 0.19 & 0.16 & 0.18 & 0.19 & 0.19 & 0.20 \\
\hline & Standard Deviation & 0.05 & 0.07 & 0.10 & 0.13 & 0.15 & 0.17 & 0.20 \\
\hline & Sharp ratio(\%) & 2.52 & 1.95 & 1.50 & 1.28 & 1.19 & 1.07 & 0.93 \\
\hline
\end{tabular}

It is clear that the sharp Ratio of the portfolio that contains Bitcoin is always higher than the one without Bitcoin. It means that bitcoin could improve the 'price' of our risk. In other words, if we want to have the same return, the risk of the portfolio with bitcoin will be lower than the portfolio without bitcoin. This has proven the ability of bitcoin to diversify the risks of the market.

\subsection{Risk analysis}

\subsubsection{Legality}

Bitcoin is a cryptocurrency and is difficult for the government to trace. Different countries have regulated Bitcoin transactions and mining. There are polarizing opinions about the regulation of Bitcoin. One of the bellwethers in the United States advocates an open attitude towards Bitcoin. The other is China, which advocates prohibiting all virtual currency transactions, including Bitcoin. Therefore, different policies caused by disparate views are the risks of Bitcoin, and it is also a topic worth discussing.

Countries have polarized attitudes towards the legitimacy of Bitcoin. Some countries like China and the United Kingdom prohibit the transaction of Bitcoin. In Chinese legislations, the trading platform shall not engage in the exchange business between legal currency and tokens or "virtual currency," shall not buy or sell tokens or "virtual currency" as a central counterparty and shall not provide pricing or information for tokens or "virtual currency" Intermediary and other services. The Bank of England recently released a report: When the market fully accepts digital currency, it will threaten 
the stability of the British financial system. However, there are other voices from some countries like the United States. From 2009 to 2017, the U.S. government's attitude towards Bitcoin has always been an open one. The U.S. Treasury Department and regulatory authorities have not made too many statements or policies to regulate Bitcoin.

As of June 2021, bitcoin was legal in the U.S., Japan, the U.K., and other developed countries. From 2009 to 2017, the U.S. government's attitude towards Bitcoin has always been an open attitude. The regulations for cryptocurrency mining in the United States are simple: if you can have cryptocurrency in a state, you can mine cryptocurrency in that state without any limitation. Differences between U.S. state regulations lead to differences in the details of mining permits of the different states. For example, New York is currently considering suspending all cryptocurrency mining operations for three years. On the other hand, Kentucky has recently passed two bills to encourage cryptocurrency mining, providing substantial property, electricity, and wage tax exemptions or tax rebates for the commercialization of cryptocurrency mining.

New York and Texas have entirely different regulations, but the states' environmental policies drive all regulations. New York State owns Greenidge Generation, one of the biggest bitcoin miners. Because more and more people are worried that mining will conflict with the state's aggressive environmental goals, the state recently introduced a bill that seeks to suspend all cryptocurrency mining for three years. Instead, Greenidge Generation proposes a plan to purchase carbon offsets to achieve carbon neutrality.

First, because China has foreign exchange control, Bitcoin's online anonymous transactions can theoretically bypass the central bank's foreign exchange defences, making foreign exchange control policy virtually useless. Bitcoin also provides the potential for money laundering transactions in an otherwise tightly controlled domestic regulatory zone. Thus, convenience has dramatically threatened the Chinese central bank's foreign exchange policy and regulatory policy. In addition, Because the RMB internationalization strategy has begun to advance fully, the essence of RMB internationalization is a regional centralized currency strategy. Nevertheless, the decentralization of Bitcoin is precisely the opposite of the Chinese RMB internationalization strategy. The collision made China's central bank have to take a "capital punishment" on Bitcoin after weighing and weighing repeatedly.

The price of Bitcoin is closely related to some of the Bitcoin policies formulated by the government. When more and more countries start to ban the mining and trading of Bitcoin, the price of Bitcoin will fall. However, when more countries show open attitudes towards Bitcoin, the price of Bitcoin will rise steadily.
So, I think that one of the most significant risks of Bitcoin is the uncertainty of the future country's policies. At the same time, Bitcoin, as a decentralized virtual currency, is contrary to the currency concept of some countries. When countries realize this problem, the future of Bitcoin will become more uncertain.

\subsubsection{Cyber risk}

In this part, we will focus on the risks that may happen in online bitcoin transactions. One prominent problem people might consider there is the risk of whether bitcoin can be stolen. That would be an incredible loss to someone who invested in cryptocurrencies. Unfortunately, in the history of bitcoin development, some events happened because of the cyber risks.

The most famous one was Mt.Gox event in June 2011. Mt. Gox was a well-known trading center of bitcoin located in Japan. The hacker got the credential of the auditor and transferred 2609 bitcoins to an address to which Mt. Gox had no keys. Therefore, Mt. Gox suspended its activities for several days. Luckily they found ways to maintain the reputation and were able to survive this time. However, a similar script acted again in 2014. At that time, Mt. Gox held $70 \%$ of the bitcoin transactions in the world. The hackers successfully caused more than 750,000BTC losses of Mt.Gox (almost 350 million dollars at that time), which directly caused Mt. Gox to announce that they were bankrupt. And none of the investors recovered their losses.

Operational risk is a significant factor for every investor to notice. A simple mistake or inadequate protection made by staff or personal can cause a tremendous loss of wealth. Take the hack event in 2011 and 2014, which caused Mt. Gox's bankruptcy as an instance; additionally, mining bitcoin is a highly high-cost project. It needs too much electricity and computing power to gain a few bitcoins, which is not worth it. There are two reasons to explain my point of view. First, bitcoin is an unreal currency that not any country made an official announcement. Also, it is a currency that could be used in the market or used to trade as traditional currency. The only value it has is just people who want to raise its price; otherwise, it is worth noting, just a single data in computers. However, there are many issues that some risk-loving investors will still decide to support bitcoin. Thereby, I also come out with some ideas to help these people to invest. Nowadays, cloud storage has become more reliable to everyone because of its convenience and more robust security than we have in the past.

\section{CONCLUSION}

In this paper, we talked about the risks that Bitcoins have faced in the current market. We start with ideas 
about whether Bitcoin will be a proper way to invest and use the MPT to determine the portfolios on the Efficient Frontier. We could find that the risks of bitcoin could be diversified with some trading assets on the actual market. Meanwhile, if we add bitcoin into our actual market portfolio, we could have a more efficient portfolio with a higher sharp Ratio. However, under extreme situations, the portfolio could only replicate the trend but will lose most of the return if we fixed relatively low volatility.

Finally, I think everyone's Bitcoin must be secured and properly controlled on this Bitcoin trading platform. Each trading platform should increase the protection of the server and increase the understanding of users. On the one hand, this is to avoid the theft of Bitcoin or the risk of Bitcoin being stolen as much as possible, and on the other hand, to minimize illegal transactions through Bitcoin. In the future, maybe the innovator of bitcoin might post an official trading centre or authoritative cooperation for their customers to use. Either in the cryptocurrency from or the currency used to buy their products. It would not be risky like it had before in the early time. At least, this idea will not hurt your wealth.

\section{REFERENCES}

[1] Wang, G., Tang, Y., Xie, C., \& Chen, S. (2019). Is bitcoin a safe haven or a hedging asset? Evidence from China. Journal of Management Science and Engineering, 4(3), 173-188. https://doi.org/10.1016/j.jmse.2019.09.001

[2] Kristoufek, L. (2020). Grandpa, grandpa, tell me the one about Bitcoin being a safe haven: Evidence from the COVID-19 pandemics. Frontiers in Physics, 8 , 296.
[3] Nakamoto, S. (2008). Bitcoin: A Peer-to-Peer Electronic Cash System. 9.

[4] Yuhanitha, N., \& Robiyanto, R. (2021). CRYPTOCURRENCIES AS A HEDGE AND SAFE HAVEN INSTRUMENTS DURING COVID-19 PANDEMIC. 18.

[5] Nguyen, T., de Bodisco, C., \& Thaver, R. (2018). FACTORS AFFECTING BITCOIN PRICE IN THE CRYPTOCURRENCY MARKET: AN EMPIRICAL STUDY. 13(1), 21. [1]Wang, G., Tang, Y., Xie, C., \& Chen, S. (2019). Is bitcoin a safe haven or a hedging asset? Evidence from China. Journal of Management Science and Engineering, 4(3), 173-188. https://doi.org/10.1016/j.jmse.2019.09.001

[6] Fabozzi, F. J., Gupta, F., \& Markowitz, H. M. (2002). The legacy of modern portfolio theory. The journal of investing, 11(3), 7-22

[7] Mangram, M. E. (2013). A simplified perspective of the Markowitz portfolio theory. Global journal of business research, 7(1), 59-70.

[8] Omisore, I., Yusuf, M., \& Christopher, N. (2011). The modern portfolio theory as an investment decision tool. Journal of Accounting and Taxation, 4(2), 19-28.

[9] Bailey, D. H., \& Lopez de Prado, M. (2012). The Sharpe ratio efficient frontier. Journal of Risk, 15(2), 13.

[10] Sharpe, W. F. (1994). The sharpe ratio. Journal of portfolio management, 21(1), 49-58.

https://doi.org/10.3389/fphy.2020.00296 